\title{
Lando Degoli
}

Sur la caractéristique de la Jacobienne des systèmes linéaires de quadriques

Czechoslovak Mathematical Journal, Vol. 36 (1986), No. 3, 476-484

Persistent URL: http://dml.cz/dmlcz/102107

\section{Terms of use:}

(C) Institute of Mathematics AS CR, 1986

Institute of Mathematics of the Czech Academy of Sciences provides access to digitized documents strictly for personal use. Each copy of any part of this document must contain these Terms of use.

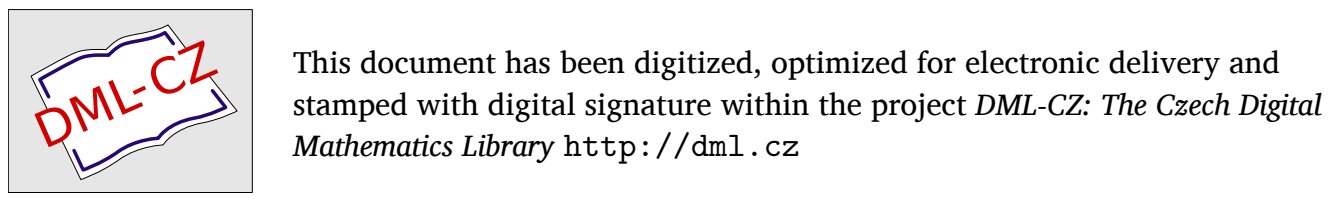




\title{
SUR LA CARACTÉRISTIQUE DE LA JACOBIENNE DES SYSTÈMES LINÉAIRES DE QUADRIQUES
}

\author{
Lando Degoli, Modena
}

(Received April 17, 1985)

Dans l'espace linéaire $S_{r}$ de coordonnées projectives homogénes $x_{i}(i=0,1,2, \ldots$ $\ldots, r$ ) choisissons $d+1$ quadriques linéairement indépendantes:

$$
f_{q}=\sum_{i, k=0}^{r} a_{q}^{i k} x_{i} x_{k} \quad(q=0,1,2, \ldots, d) .
$$

Le système linéaire $L_{d}$ de dimension $d$, qui en résulte, est exprimé par l'equation:

$$
\sum_{q=0}^{d} \mu_{q} f_{q}=0 \text {. }
$$

Supposons que la matrice Jacobienne à $r+1$ lignes et $d+1$ colonnes:

$$
J=\left\|\frac{\partial f_{q}}{\partial x_{i}}\right\| \quad\left(\begin{array}{l}
q=0,1,2, \ldots, d \\
i=0,1,2, \ldots, r
\end{array}\right)
$$

soit à caractéristique $m \leqq d$.

Si la caractéristique de la Jacobienne est $m=r-h(h \geqq 0)$, un point générique de $S_{r}$ est conjugué avec un $S_{h}$ par rapport à toutes les quadriques du système.

Un système $L_{d / m}$ de dimension $d$ et caractéristique $m$ possède $d+1$ quadriques linéairement indépendantes, parmi les quelles on peut choisir $m$ quadriques fonctionellement indépendantes, desquelles toutes les autres dépendent.

Un système $L_{d / m}(m \leqq d)$, qui ne possède pas de systèmes subordonnés essentiels $L_{g / c}$ (voir: [6]), avec:

$$
2 \leqq g \leqq d-1,2 \leqq c \leqq m-1, c \leqq g
$$

est dit irréductible de prémier espèce.

Nous avons démontré (voir: [6]) le:

Théorème A. La condition nécessaire et suffisante pour qu'un système linéaire irréductible de prémière espèce soit à Jacobienne de caractéristique $r-k \leqq d$ $(k \geqq 0)$ c'est que les quadriques du système, qui passent par un point générique de $S_{r}$, possédent en commun un $S_{k+1}$.

Donnés deux systèmes linéaires $L_{a}$ et $L_{b}$, qui ont en commun un système linéaire $L_{c}$, leur système-union résulte de dimension: $a+b-c$. 
Nous dirons le système $L_{d / m}(m \leqq d)$ réductible, s'il est l'union des systèmes subordonnés, parmi lesquels un au moins: $L_{a / b}(b \leqq a)$ n'a pas des quadriques en commun avec les autres.

Nous dirons que les systèmes linéaires $L_{d_{1}}, L_{d_{2}}, \ldots, L_{d_{s}}$ forment une chaine quand ils ne sont pas réductibles et si $L_{d_{1}}$ et $L_{d_{2}}$ ont en commun une quadrique au moins, leur système union $L_{a}$ a en commun avec $L_{d_{3}}$ au moins une quadrique, et le système $L_{b}$ union de $L_{a}$ avec $L_{d_{3}}$ a en commun au moins une quadrique avec $L_{d_{4}}$ et ainsi de suite jusqu'à $L_{d_{s}}$.

Il existe le:

Lemme 1. Si le système $L_{d}$ possède une chaine de systèmes subordonnés $L_{d_{1}}, L_{d_{2}}, \ldots$ $\ldots, L_{d_{s}}$ et pas d'autres quadriques fonctionellement indépendantes, il est possible choisir parmi les quadriques de $L_{d_{1}}, L_{d_{2}}, \ldots, L_{d_{s}}$, qui passent par un point $P$ de $S_{r}$, $d$ quadriques linéairement indépendantes, lesquelles individualisent le système $L_{d-1}$ de quadriques de $L_{d}$, qui passent par $P$.

Les équations de $L_{d_{1}}$ et $L_{d_{2}}$ résultent:

$$
\begin{aligned}
& \mu_{0} f_{0}+\mu_{1} f_{1}+\ldots+\mu_{h} f_{h}+\ldots+\mu_{d_{1}} f_{d_{1}}=0, \\
& \xi_{0} g_{0}+\xi_{1} g_{1}+\ldots+\xi_{h} g_{h}+\ldots+\xi_{d_{2}} g_{d_{2}}=0 .
\end{aligned}
$$

L'équation du système-union $L_{d}$ devient:

$$
\begin{gathered}
v_{0} f_{0}+v_{1} f_{1}+\ldots+v_{h} f_{h}+\ldots+v_{d_{1}} f_{d_{1}}+ \\
+\theta_{0} g_{0}+\theta_{1} g_{1}+\ldots+\theta_{h} g_{h}+\ldots+\theta_{d_{2}} g_{d_{2}}=0 .
\end{gathered}
$$

Puisque $L_{d_{1}}$ et $L_{d_{2}}$ ont en commun au moins une quadrique, nous pouvons supposer $f_{h}$ coincident avec $g_{h}$.

Soit $P$ un point de $S_{r}$. Indiquons $f_{0}(P), f_{1}(P), \ldots, g_{0}(P), g_{1}(P) \ldots$ les valeurs complexes des quadriques en $P$. En remplaçant les coordonnées de $P$ dans $(1)$ on peut déterminer $\mu_{h}$ et $\xi_{h}$ en fonction des $\mu$ et $\xi$ restants.

On obtient:

$$
\begin{aligned}
& \mu_{0}\left(f_{0}+\frac{f_{0}(P)}{f_{h}(P)} f_{h}\right)+\mu_{1}\left(f_{1}+\frac{f_{1}(P)}{f_{h}(P)} f_{h}\right)+\ldots+\mu_{d_{1}}\left(f_{d_{1}}+\frac{f_{d_{1}}(P)}{f_{h}(P)} f_{h}\right)=0, \\
& \xi_{0}\left(g_{0}+\frac{g_{0}(P)}{f_{h}(P)} f_{h}\right)+\xi_{1}\left(g_{1}+\frac{g_{1}(P)}{f_{h}(P)} f_{h}\right)+\ldots+\xi_{d_{2}}\left(g_{d_{2}}+\frac{g_{d_{2}}(P)}{f_{h}(P)} f_{h}\right)=0 .
\end{aligned}
$$

Dans ces systèmes $L_{d_{1}-1}$ et $L_{d_{2}-1}$ manquent les paramètres $\mu_{h}$ et $\xi_{h}$. En opérant de même façon dans le système $(2)$ et en éliminant le paramètre $\left(v_{h}+\theta_{h}\right)$ on obtient:

$$
\begin{gathered}
v_{0}\left(f_{0}+\frac{f_{0}(P)}{f_{h}(P)} f_{h}\right)+v_{1}\left(f_{1}+\frac{f_{1}(P)}{f_{h}(P)} f_{h}\right)+\ldots+v_{d_{1}}\left(f_{d_{1}}+\frac{f_{d_{1}}(P)}{f_{h}(P)} f_{h}\right)+ \\
+\theta_{0}\left(g_{0}+\frac{g_{0}(P)}{f_{h}(P)} f_{h}\right)+\theta_{1}\left(g_{1}+\frac{\iota_{1}(P)}{f_{h}(P)} f_{h}\right)+\ldots+\theta_{d_{2}}\left(g_{d_{2}}+\frac{g_{d_{2}}(P)}{f_{h}(P)} f_{h}\right)=0 .
\end{gathered}
$$


Puisque le système linéaire (4) est individualisé par les mêmes quadriques linéairement indépendantes des systèmes (3), il en résulte que le système $L_{d-1}$ des quadriques de $L_{d}$, qui passent par $P$, est individualisé par a quadriques linéairement indépendantes de $L_{d_{1}-1}$ et $L_{d_{2}-1}$.

Mais par hypothèse les systèmes subordonnés de $L_{d}$ forment une chaine. Donc le système-union $L_{a}$ possède en commun avec $L_{d_{3}}$ au moins une quadrique.

En opérant de même façon on prouvera que le système $L_{b-1}$ de quadriques de $L_{b}$, système-union de $L_{a}$ avec $L_{d_{3}}$, qui passent par $P$, est individualisé par $b$ quadriques linéairement indépendantes extraites de $L_{a-1}$ et $L_{d_{3}-1}$, c'est à dire de: $L_{d_{1}-1}, L_{d_{2}-1}$, $L_{d_{3}-1}$.

En continuant de cette manière, puisque $L_{d}$ ne possède pas d'autres quadriques fonctionellement indépendantes, le lemme résulte démontré.

Il existe le:

Théorème B. La condition nécessaire et suffisante pour que le système linéaire de quadriques $L_{d}$, qui possède une chaine de systèmes irréductibles de prémière espéce $L_{d_{1} / m_{1}}, L_{d_{2} / m_{2}}, \ldots, L_{d_{s} / m_{s}}\left(m_{i} \leqq d_{i} ; i=1,2, \ldots, s\right)$ et pas d'autres quadriques fonctionellement indépendantes, soit à Jacobienne de caractéristique $r-k \leqq d$ $(k \geqq 0)$, c'est que les quadriques $d u$ système qui passent par un point quelconque de $S_{r}$ possèdent en commun un $S_{k+1}$.

Supposons d'abord que la caractéristique de la Jacobienne soit:

$$
r-k \leqq d, \quad(k \geqq 0) .
$$

Cela signifie que parmi le $d+1$ quadriques linéairement indépendantes qu'individualisent $L_{d}$ il y a $r-k$ quadriques fonctionellement indépendantes.

Puisque $L_{d_{1}}$ est un système irréductible de prémière espèce, il satisfait au théorème A. Donc les quadriques de $L_{d_{1}}$, qui passent par un point générique $P$ de $S_{r}$ ont en commun un $S_{r-m_{1}+1}$. Elles constituent le système $L_{d_{1}-1}$.

Considérons le système $L_{d-1}$ de quadriques de $L_{d}$, qui passent par $P$. Les quadriques fonctionellement indépendantes de ce système, qui n'appartient pas à $L_{d_{1}-1}$ sont $r-k-m_{1}$.

En effet soient:

$$
\begin{aligned}
& \mu_{0} f_{0}+\mu_{1} f_{1}+\ldots+\mu_{d_{1}} f_{d_{1}}=0, \\
& \xi_{0} f_{0}+\xi_{1} f_{1}+\ldots+\xi_{d_{1}} f_{d_{1}}+\xi_{d_{1}+1} f_{d_{1}+1}+\ldots+\xi_{d} f_{d}=0
\end{aligned}
$$

les équations respectives de $L_{d_{1}}$ et $L_{d}$.

Si nous imposons à tout les deux systèmes de passer par $P$, en éliminant $\mu_{0}$ et $\xi_{0}$ on obtient.

$$
\mu_{1}\left(f_{1}+\frac{f_{1}(P)}{f_{0}(P)} f_{0}\right)+\mu_{2}\left(f_{0}+\frac{\left.f_{2}^{\prime} P\right)}{f_{0}(P)} f_{0}\right)+\ldots+\mu_{d_{1}}\left(f_{d_{1}}+\frac{f_{d_{1}}(P)}{f_{0}(P)} f_{0}\right)=0,
$$




$$
\begin{gathered}
\xi_{1}\left(f_{1}+\frac{f_{1}(P)}{f_{0}(P)} f_{0}\right)+\xi_{2}\left(f_{0}+\frac{f_{2}(P)}{f_{0}(P)} f_{0}\right)+\ldots+\xi_{d_{1}}\left(f_{d_{1}}+\frac{f_{d_{1}}(P)}{f_{0}(P)} f_{0}\right)+ \\
+\xi_{d_{1}+1}\left(f_{d_{1}+1}+\frac{f_{d_{1}}(P)}{f_{0}(P)} f_{0}\right)+\ldots+\xi_{d}\left(f_{d}+\frac{f_{d}(P)}{f_{0}(P)} f_{0}\right)=0 .
\end{gathered}
$$

Le nombre de quadriques, fonctionellement indépendantes de $L_{d}$ qui n'appartiennent pas à $L_{d_{1}}$, c'est la difference des caractéristiques; c'est à dire: $r-k-m_{1}$.

$\mathrm{Ma}(5)$ et (6) prouvent que ce nombre reste invarié en passant à $L_{d-1}$ et $L_{d_{1}-1}$ parce que les quadriques, qui individualisent $L_{d_{1}-1}$, se trouvent toutes dans $L_{d-1}$.

Les hyperplans polaires des $r-k-m_{1}$ quadriques fonctionellement indépendantes de $L_{d-1}$, qui n'appartiennent pas à $L_{d_{1}-1}$, passent tous par $P$. Ils coupent l' $S_{r-m_{1}+1}$ selon un $S_{k+1}$, qui résulte au moins tangente à toutes les quadriques de $L_{d-1}$.

Ce résultat est donc indépendant de $d_{1}$ et de $m_{1}$.

En opérant de même manière sur $L_{d_{2} / m_{2}}, L_{d_{3} / m_{3}}$ etc. jusqu'à $L_{d_{s} / m_{s}}$ on obtiendra les espaces:

$$
S_{r-m_{2}+1}, S_{r-m_{3}+1}, \ldots, S_{r-m_{s}+1}
$$

lesquels, entrecoupés par les hyperplans polaires des restantes quadriques fonctionellement indépendantes de $L_{d-1}$, donnent toujours le même $S_{k+1}$.

Pour cela toutes les quadriques de $L_{d_{1}-1}, L_{d_{2}-1}, \ldots, L_{d_{s}-1}$ ont en commun le precédent $S_{k+1}$, parce qu'il est conténu dans les:

précédents.

$$
S_{r-m_{1}+1}, S_{r-m_{2}+1}, \ldots, S_{r-m_{s}+1} .
$$

Mais puisque $L_{d_{1}}, L_{d_{2}}, \ldots, L_{d_{s}}$ forment une chaine dans $L_{d}$ et ce système ne possède pas d'autres quadriques fonctionellement indépendantes, il est toujour possible à cause du lemme 1 , choisir dans $L_{d_{1}-1}, L_{d_{2}-1}, \ldots, L_{d_{s}-1}, d$ quadriques linéairement indépendantes, qui individualisent $L_{d-1}$.

Mais ces $d$ quadriques ont en commun $S_{k+1}$, il en résulte que toutes les quadriques de $L_{d-1}$ possèdent en commun $S_{k+1}$, comme il fallait démontrer.

Viceversa si toutes les quadriques de $L_{d}$, qui passent par un point $P$, ont en commun un $S_{k+1}$, il est évident que le point $P$ a pour conjugué le même $S_{k+1}$ par rapport à toutes les quadriques, qui passent par $P$.

Une quadrique $G$ de $L_{d}$, qui ne passe pas par $P$, ne contient pas $S_{k+1}$. L'hyperplan polaire de $G$ par rapport à $P$ ne contient pas $S_{k+1}$, autrement dît $P$ appartiendrai à la quadrique.

Pour cela l'hyperplan coupera $S_{k+1}$ selon $S_{k}$ par rapport à toutes les quadriques de $L_{d}$. Cela signifie que la Jacobienne de $L_{d}$ est à caractéristique $r-k$, ce qu'il fallait démontrer. 
Remarque. Le théorème est valable aussi dans l'hypothèse que $L_{d_{1} / m_{1}}, L_{d_{2} / m_{2}}, \ldots$ $\ldots, L_{d_{s} / m_{s}}\left(m_{i} \leqq d_{i}, i=1,2, \ldots, s\right)$ soient des systèmes analogues à $L_{d}$, qui satisfont au théorème $B$.

En effet $L_{d}$, par le théorème démontré juît de la même propriété des systèmes irréductibles de prémière espèce et pour cela rien change dans la démonstration précedente.

Il s'ensuit que le théorème existe aussi dans l'hypothèse que $L_{d}$ possède une chaine de systèmes linéaires $L_{d_{i} / m_{i}}\left(m_{i} \leqq d_{i}, i=1,2, \ldots, s\right)$ quelconques, à condition qu'il ne possède pas d'autres quadriques fonctionellement indépendantes.

Nous dirons systèmes irréductibles de seconde espèce, les systèmes linéaires de quadriques, qui satisfont aux hypothèses du théorème $B$ avec les considérations de la Remarque précedente.

Il existe le:

Lemme 2. Considérons un système $L_{d / m}(m \leqq d)$ de $S_{r}$, qui possède seulement des systèmes subordonnés $L_{d_{1} / m_{1}}, L_{d_{2} / m_{2}}, \ldots, L_{d_{s} / m_{s}}\left(m_{i} \leqq d_{i}, i=1,2, \ldots, s\right)$ irréductibles (de prémière on de seconde espèce), qui ne s'entrecoupent pas et éventuellement $p(p \geqq 0)$ quadriques fonctionellement indépendantes.

Il en résulte:

$$
\begin{gathered}
d=d_{1}+d_{2}+\ldots+d_{s}+s+p-1, \\
m=m_{1}+m_{2}+\ldots+m_{s}+p .
\end{gathered}
$$

En effet, $L_{d}$ résulte le système-union des systèmes donnés et des $p(p \geqq 0)$ quadriques fonctionellement indépendantes, et pour cela sa dimension augmentée de l'unité sera égal à la somme des dimensions des systèmes subordonnés augmentés de l'unité, et du nombre de quadriques fonctionellement indépendantes.

On aura:

$$
d+1=\left(d_{1}+1\right)+\left(d_{2}+1\right)+\ldots+\left(d_{s}+1\right)+p .
$$

C'est a dire:

$$
d=d_{1}+d_{2}+\ldots+d_{s}+s+p-1 .
$$

En outre les quadriques fonctionellement indépendantes parmi les $d+1$ linéairement indépendantes, qui individualisent $L_{d}$, sont: $m$.

Les quadriques fonctionellement indépendantes de chacun système sont toujours diverses des quadriques des autres systèmes, car les systèmes n'ont pas des quadriques en commun. Il s'ensuit que le nombre des quadriques fonctionellement indépendantes de tous les systèmes subordonnés est donné par la somme des caractéristiques:

$$
m_{1}+m_{2}+\ldots+m_{s} \text {. }
$$

Ce nombre augmenté de $p$, c'est à dire du nombre des quadriques fonctionellement indépendantes externes aux systèmes, donnera le nombre total des quadriques 
fonctionellement indépendantes de $L_{d}$. Il en résulte:

$$
m=m_{1}+m_{2}+\ldots+m_{s}+p,
$$

comme il fallait prouver.

Il éxiste le:

Théorème C. Condition nécessaire et suffisante pour qu'un système linéaire $L_{d}$ de quadriques de $S_{r}$, qui possède les systèmes subordonnés irréductibles (de prémière ou de seconde espèce):

$$
L_{d_{1} / m_{1}}, L_{d_{2} / m_{2}}, \ldots, L_{d_{s} / m_{s}} \quad\left(m_{i} \leqq d_{i}, i=1,2, \ldots, s\right) .
$$

tels que les quadriques d'un système n'appartiennent pas aux autres, et eventuellement $p(p \geqq 0)$ quadriques fonctionellement indépendantes, soit à Jacobienne de caractéristique $r-k \leqq d$, c'est que les quadriques des systèmes subordonnés, qui passent par un point génerique de $S_{r}$ constituent un $L_{d-s-p}$, et possèdent en commun un $S_{k+s+p}$.

Puisque les systèmes subordonnés satisfont l'hypothèse du lemme 2, il s'ensuit:

$$
\begin{aligned}
& d=d_{1}+d_{2}+\ldots+d_{s}+s+p-1 \\
& r-k=m_{1}+m_{2}+\ldots+m_{s}+p .
\end{aligned}
$$

Puisque les systèmes: $L_{d_{1} / m_{1}}, L_{d_{2} / m_{2}}, \ldots, L_{d_{s} / m_{s}}$ sont irréductibles de prémière ou de seconde espèce ils satisfont au théorème $A$, ou bien au théorème $B$.

Dans les deux cas par un point $P$ de $S_{r}$ il passe respectivement un:

$$
L_{d_{1}-1}, L_{d_{2}-1}, \ldots, L_{d_{s}-1}
$$

de quadriques, qui ont en commun respectivement un:

$$
S_{r-m_{1}+1}, S_{r-m_{2}+1}, \ldots, S_{r-m_{s}+1} \text {. }
$$

Ces espaces par (7) se coupent dans un:

$$
S_{r-m_{1}-m_{2}-\cdots-m_{s}+s}=S_{k+s+p} .
$$

Mais puisque on peut choisir en chacun des systèmes, $d_{1}, d_{2}, \ldots, d_{s}$ quadriques linéairement indépendantes, on trouve que les quadriques de ces systèmes, qui passent par $P$ forment par (8) un système:

$$
L_{d_{1}+d_{2}+\cdots+d_{s}-1}=L_{d-s-p},
$$

dont les quadriques ont en commun $S_{k+s+p}$, comme il fallait demontrer.

La condition est donc nécessaire.

Elle est aussi suffisante.

En effet dans les hypothèse du théorème, supposons que par un point $P$ de $S_{r}$ 
il passe un système $L_{d-s-p}$, individualisé par des quadriques, qui appartiennent à:

$$
L_{d_{1} / m_{1}}, L_{d_{2} / m_{2}}, \ldots, L_{d_{s} / m_{s}}
$$

et qui possèdent en commun un $S_{k+s+p}$.

Notons $x$ la caractéristique de $L_{d}$.

Pour le lemme 2 il s'ensuit:

$$
\begin{aligned}
& d=d_{1}+d_{2}+\ldots+d_{s}+s+p-1, \\
& x=m_{1}+m_{2}+\ldots+m_{s}+p .
\end{aligned}
$$

Puisque les systèmes subordonnés sont irréductibles par un point générique $P$ de $S_{r}$ il passe respectivement un:

$$
L_{d_{1}-1}, L_{d_{2}-1}, \ldots, L_{d_{s}-1}
$$

de quadriques, qui possèdent en commun respectivement un:

$$
S_{r-m_{1}+1}, S_{r-m_{2}+1}, \ldots, S_{r-m_{s}+1} \text {. }
$$

Ces espaces par (9) s'entrecoupent dans:

$$
S_{r-m_{1}-m_{2}-\cdots-m_{s}+s}=S_{r-x+s+p} .
$$

Mais de ces systèmes on peut extraire respectivement $d_{1}, d_{2}, \ldots, d_{s}$ quadriques linéairement indépendantes, qui individualisent le système:

$$
L_{d_{1}+d_{2}+\cdots+d_{s}-1}=L_{d-s-p}
$$

de quadriques, qui ont en commun $S_{r-x+s+p}$.

Les quadriques, qui individualisent $L_{d-s-p}$ sont toutes quadriques linéairement indépendantes, qui appartiennent aux systèmes subordonnés et qui passent par $P$. Mais telles quadriques possèdent en commun par hypothèse $S_{k+s+p}$, qui devra coincider avec $S_{r-x+s+p}$.

Il s'ensuit $x=r-k$, comme il fallait démontrer.

Les systèmes $L_{d / m}(m \leqq d)$ irréducibles de prémière et seconde espèce ne sont pas les seuls à jouir des propriétés exprimées par les théorèmes $\mathrm{A}$ et $\mathrm{B}$.

Il existe le:

Théorème D. Condition nécessaire et suffisante pour que les quadriques d'un système linéaire $L_{d}$, qui passent par un point $P$ de $S_{r}$, possèdent en commun un $S_{k+1}$ c'est que $\infty^{\grave{k}}$ cordes de la variété base du système donné sortent de P, en constituant un $S_{k+1}$.

Soit un système linéaire $L_{d}$ tel que toutes les quadriques du système, qui passent par un point générique $P$, possèdent en commun un $S_{k+1}$. Les quadriques individualisent un système $L_{d-1}$. Une ultérieure quadrique de $L_{d}$, qui n'appartient pas à $L_{d-1}$, entrecoupe $S_{k+1}$ selon une quadrique $G_{k}$ de $S_{k+1}$, qui résulte commune à toutes les quadriques de système donné et par conséquent elle appartient à sa variété base $V$. 
Une droite générique de $S_{k+1}$, qui passe par $P$ entrecoupe $G_{k}$ en deux points, qui appartiennent a $V$. Il s'ensuit qu'elle est une corde de $V$.

Puisque on peut répéter ce raisonnement pour toutes les droites de $S_{k+1}$, qui sortent de $P$, il s'ensuit que par $P$ passent $\infty^{k}$ cordes de $V$.

Viceversa si $\infty^{k}$ cordes de $V$, variété base du système $L_{d}$, passent par un point générique $P$ et constituent un $S_{k+1}$, une corde générique $s$, choisie entr'elles, aura deux points $R$ et $T$ sur $V$ et pour cela sur toutes les quadriques de $L_{d}$.

Mais les quadriques qui passent par $P$, forment un système $L_{d-1}$, dans le quel chaque quadrique possède trois points $P, R, S$ de la droite $s$. Donc toute la droite appartiendra à toutes ces quadriques.

Et puisque on peut répéter tel raisonnement pour les $\infty^{k}$ cordes de $V$, il en résulte que les quadriques de $L_{d-1}$ possèdent en commun un $S_{k+1}$.

Il s'ensuit que:

Si les quadriques de $L_{d}$, qui passent par $P$, ont en commun seulement $S_{k+1}$, en excluant pour cela qu'elles possèdent en commun un $S_{h}(h \geqq 1)$ tangente en $P$, la caractéristique de la Jacobienne de $L_{d}$ est: $r-k$.

En effet notons $Q$ le conjugué de $\mathrm{P}$ par rapport à $R$ et $T$. Il résulte le conjugué de $P$ par rapport à toutes les quadriques du système.

On peut dire la même chose pour chacune des $\infty^{k}$ cordes et seulement pour elles. Donc $P$ résulte le conjugué $\mathrm{d}^{\prime} \infty^{k}$ points qui constituent une variété à $k$ dimensions.

Celle-ci ne peut pas être qu'un $S_{k}$.

En effet elle doit résulter l'intersection des hyperplans polaires de $P$ par rapport à chaque quadrique du système.

Il s'ensuit que $P$ à pour conjugué un $S_{k}$ et que la caractéristique de la Jacobienne est: $r-k$.

Nous dirons: système à variété base $\infty^{k}$ un système linéaire de quadrique, qui satisfait au théorème $D$.

Ce type de système renferme, tous les systèmes irréductibles de prémière et seconde espèce à cause des théorèmes $\mathrm{A}$ at $\mathrm{B}$.

Mais il y a d'autres systèmes de ce type.

Considerons, par example, le système linéaire individualisée par les quadriques, qu'on déduit des équations:

$$
\frac{x_{0}}{x_{1}}=\frac{x_{1}}{x_{2}}=\frac{x_{2}}{x_{3}}
$$

c'est à dire:

$$
\mu_{0}\left(x_{0} x_{2}-x_{1}^{2}\right)+\mu_{1}\left(x_{0} x_{3}-x_{1} x_{2}\right)+\mu_{2}\left(x_{1} x_{3}-x_{2}^{2}\right)=0 .
$$

Puisque les trois quadriques sont fonctionellement indépendantes, le système résulte un $L_{2 / 3}$ de $S_{3}$, évidemment sans systèmes subordonnés essentiels, qui toutefois ne satisfait pas à la condition $r-k \leqq d$ du théorème $\mathrm{A}$. 
Mais sa variété base est la cubique de $S_{3}$, d'equations parametriques:

$$
x_{0}=1, \quad x_{1}=\lambda, \quad x_{2}=\lambda^{2}, \quad x_{3}=\lambda^{3} .
$$

Ses cordes remplissent tout $S_{3}$.

Il s'ensuit que tel système est à variété base $\infty^{0}$ et que par un point génerique de $S_{3}$ passent $\infty^{1}$ quadriques du système, qui possèdent en commun une droite.

\section{Bibliographie}

[1] F. Palatini: Sulle superficie algebriche $i$ cui $S_{h}(h+1)$ secanti non riempiono lo spazio ambiente. Atti R. Acc. Torino 41, (1906).

[2] G. Bonferroni: Sui sistemi lineari di quadriche la cui jacobiana ha dimensione irregolare. R. Acc. Scienze Torino vol. 50, (1914-1915).

[3] A. Terracini: Alcune questioni sugli spazi tangenti e osculatori ad una varietà. Atti. R. Acc. Sc. Torino. Nota II, 51, (1916) III, 55, (1919-1920).

[4] L. Muracchini: Sulle varietà $V_{5}$ i cui spazi tangenti ricoprono una varietà $W$ di dimensione inferiore all'ordinaria. (parte II) Riv. Mat. Univ. di Parma, 3, (1952), 75-89.

[5] S. Xambo: On projectives varieties of minimal degree. Collectanea Mathematica, Barcelona, vol. $X X X I I,(1981)$.

[6] L. Degoli: Un théorème sur les systèmes linéaires de quadriques à Jacobienne indeterminée. Studia Scientiarum Mathematicarum Hungarica Budapest. Tomo 17, (1982), 325-330.

[7] L. Degoli: Due nuovi teoremi sui sistemi lineari di quadriche a Jacobiana identicamente nulla. Collectanea Mathematica, Barcelona, vol. XXXIII, (1982).

[8] L. Degoli: Trois nouveaux théorèmes sur les systèmes linéaires de quadriques à Jacobienne identiquement nulle. Demonstratio Mathematica, Warszawa, Vol. 16, (1983).

[9] L. Degoli: Alcuni teoremi sui sistemi lineari di quadriche a Jacobiana identicamente nulla. Revue d'analyse numérique et de théorie de l'approximation - Cluj-Napoca. Tome 26 (49), (1984), 33-43.

Adresse: Via Berengario no 82/C, 41012 Carpi (Modena), Italy. 